\title{
On the Localization Algorithm of Wireless Sensor Network and Its Application
}

\author{
https://doi.org/10.3991/ijoe.v13i03.6858 \\ Honglei Jia \\ Jilin University, Changchun, China \\ jiahl@vip.163.com \\ Jiaxin Zheng \\ Jilin University, Changchun, China \\ zhengjiaxin_0628@126.com \\ Gang Wang * \\ Jilin University, Changchun, China \\ wangganggoodman@ $126 . \mathrm{com}$ \\ Yulong Chen \\ Jilin University, Changchun, China \\ $1097402292 @ q q . c o m$ \\ Dongyan Huang \\ Jilin University, Changchun, China \\ cchdy760829@sina.com \\ Hongfang Yuan \\ Jilin University, Changchun, China
}

\begin{abstract}
This paper carries out in-depth and meticulous analysis of the DV-Hop localization algorithm for wireless sensor network. It improves the DV-Hop algorithm into a node localization algorithm based on one-hop range, and proposes the centroid particle swarm optimization localization algorithm based on RSSI by adding the RSSI and particle swarm optimization algorithm to the traditional centroid localization algorithm. Simulation experiment proves that the two algorithms have excellent effect.
\end{abstract}

Keywords-DV-Hoplocalization algorithm; WSN localization; one-hop; RSSI; particle swarm optimization algorithm.

\section{Introduction}

The wireless sensor network (WSN) is a data communication network built on the integration of various new communication technologies and devices [1]. It is very difficult to control the WSN because it requires a lot of sensors to complete complex 
information processing and data operations [2-4]. However, thanks to the outstanding performance, it has enjoyed great popularity in the field of network communication. The network is capable of acquiring a gigantic amount of information from all aspects. With the large number and various types of sensor nodes, it satisfies all kinds of information needs [5]. In the WSN, the independent wireless sensor nodes have limited ability to detect, send and receive information. However, when multiple sensor nodes are combined into one, the WSN would have an increasingly stronger capability of information detection, transmission and reception[6]. In order to control the numerous sensor nodes, it is necessary to set up a special network protocol. With the protocol in place, it is possible to manage and control the data acquisition and processing of the nodes, and to carry out monitoring and information identification of all kinds of information within the radiation of the network, thereby fulfilling the corresponding monitoring purposes [7-8].

As is known to all, computer communication undergoes a significant change in computing model every 15 years [9]. The pattern applies to virtually every country in the world although it is based only on the experience of developed countries. Driven by computer communication technology, the human society is rapidly entering the Internet era[10]. The invention and extensive use of the Internet have already brought unprecedented changes to the economic, politics and social conditions of the world. As a result, almost all countries across the globe are stepping up the investment on information infrastructure [11-12]. Many new computer networks start to appear in the investment craze. Among them, the fast growing one is the WSN. The network is, in essence, an Internet of Things that links up sensors, water conservancy networks, power grid, road networks, communication networks, and pipe networks. At the dawn of the ear of the Internet of Things, the WSN is having an immense impact to people's living and working environments [13]. With the further development and maturation of relevant technologies, it can be expected that the WSN would be deeply applied to every aspect of the social life.

\section{The structure of WSN}

The main components of WSN are sensor nodes, sink nodes and management nodes. The network links up the user, the detection target, and the sensor nodes in an efficient manner, making it easier to detect the target [14].

Figure 1 illustrates the specific structure of the network. There are many sensor nodes in the monitoring area, which are combined in a certain way to synergically detect the target. After detecting the relevant information of the target object, the nodes pre-process the information and send the pre-processed information to the sink nodes. Then, the sink nodes send the received information to the control terminal. The user can communicate with the sink nodes through the computer network. Upon receiving the monitoring request, the sink nodes send a command to the sensor nodes, asking the latter to transmit the acquired data to the sink nodes [15-16].

In the network, the most basic units are the sensor network nodes, which can be further divided into units like processor, sensor, A/D converter, power supply, etc. See Figure 2 for more details. 


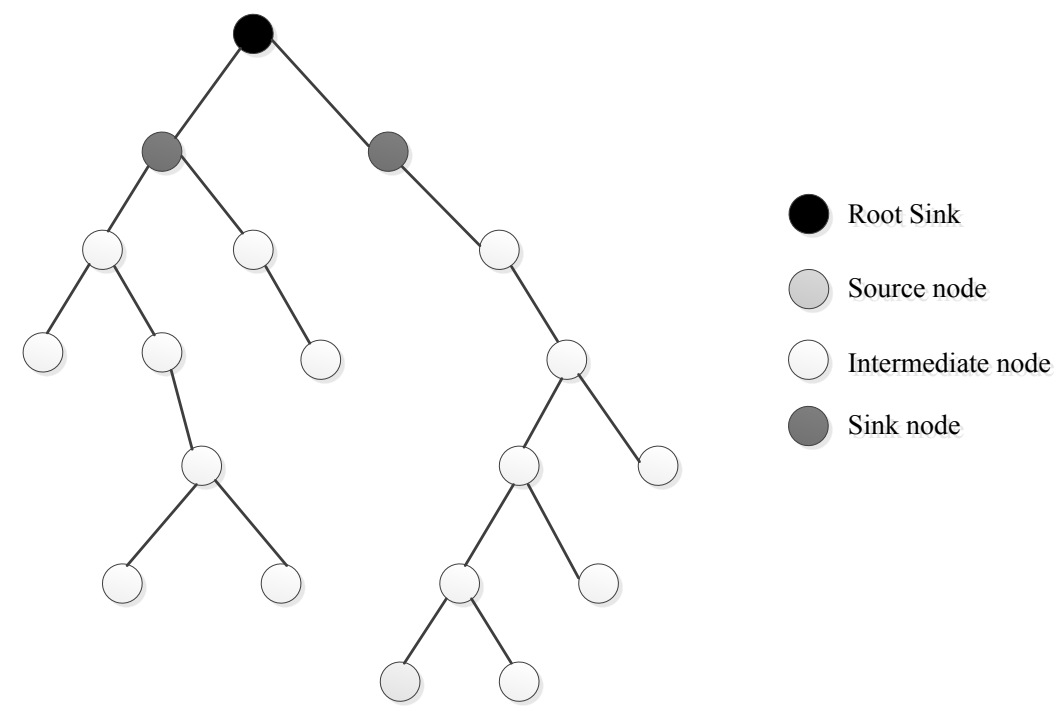

Fig. 1. The structure of WSN

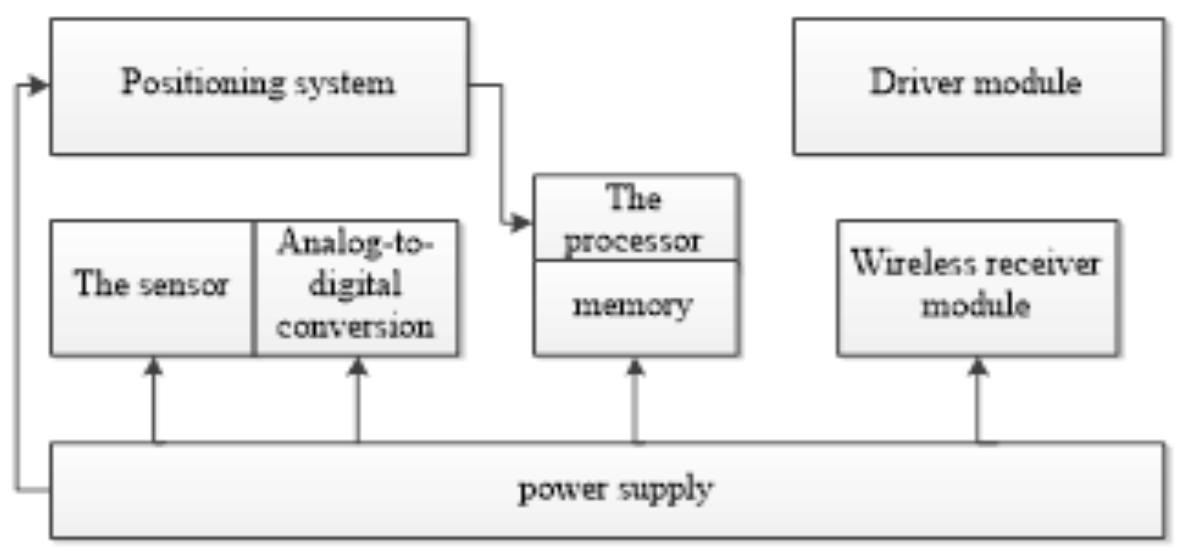

Fig. 2. The hardware structure of WSN

\section{The improved DV-Hop algorithm}

\subsection{The design of node structure}

The structure of WSN must be carefully designed for it has a significant effect on the processing performance of the whole network [17]. In the traditional DV-Hop algorithm, the location information of the anchor node broadcast is in the format of $\left\{i d, x_{i}, y_{i}, H_{o p s}\right\}$, where the variables respectively represent the number, coordinates and the current hop count of the anchor node [18]. The format is mainly for the anchor node. If the unknown node is already localized, this format is not often used. 
In the improved algorithm, however, each node has its corresponding information structure. If the node is unknown, the corresponding information structure is $\left\{i d, x_{i}, y_{i}\right.$, $\left.H_{o p s}\right\}$. The only difference from the traditional format is the addition of the variable $A_{\text {id }}$. The variable has multiple values. If the value is 0 , the anchor node can be determined as a level 1 anchor node, i.e. embedded in the system. If the value is 1 , the anchor node can be regarded as a level 2 anchor node, which is transformed from a localized unknown node. If the value is 2 , the anchor node should be categorized as a level 3 anchor node although it can also be converted from an unknown node. To minimize the localization error, researchers generally choose advanced nodes to locate the unknown node. The format of the information on the unknown node contains two parts: the former part is the broadcast information, including the node number, coordinates, etc., and the latter part is the variable depicting how the unknown node used to receive and store the data packets from the anchor node. In short, the information should be expressed in the following format: $\left\{i, A i, x i, y i, H_{o p s}\right\}$. The format contains the corresponding incident angle value, the coordinates of the node, and the variable of the information.

\subsection{The localization of unknown node}

(1) When all anchor nodes in the network are in the broadcast state, the anchor nodes send data packets on their location information to all the unknown nodes within their communication coverage. The data packets are in the format of $\{i, A i, x i, y i$, $H_{o p s}$ id, Di\}. id stands for the number assigned to an anchor node. Each node has a unique identification number. That is because the nodes in the network have no inherent order, making it possible to encode the entire network in numbers. The id value is pre-set and should not be changed. The coordinates of the anchor node is obtained by GPS tools, which are highly accurate. The judgement should be make after the information sent by the anchor node reaches the unknown node. The value of Hops $\mathrm{id}_{\mathrm{id}}$ provides the basis for judgement. If the value equals 1 , save the received data packet; if it is not 1 , discard the received data packet. As the nodes in WSN are not configured regularly, the one-hop range of an unknown node is not definite. In this case, as long as a packet of $\mathrm{Hops}_{\mathrm{id}}=1$ is received, the node is in the suspended state and no longer receives any more packet. During data reception, the unknown node also judges the $\mathrm{A}_{\mathrm{id}}$ of the data packet and makes choices based on the priorities. If a data packet has a high priority and the corresponding Hops $_{\mathrm{id}}=1$, it would be saved while the data packets of low priority would be discarded. However, if the received data packet has a highly prioritized $\mathrm{A}_{\mathrm{id}}$, but the hop count is not 1 , it would not be saved earlier than the data packets with Hops $_{i d}=1$. The hop count is prioritized because the analysis in this paper is based on the localization algorithm within the one-hop range. The initial value of $A_{i d}$ is either 0 or 1 . If it is 0 , the corresponding node should be regarded as an unknown node, which cannot send data packets. Thus, if $A_{\text {id }}$ is 0 , the node can participate in the localization of other nodes.

(2) After the unknown node receives the data packet, it firstly saves the value. If the packet is judged as available, the node would enter the suspended state and no longer receives data packet. The data packet information tells about the anchor nodes 
within the one-hop range of the unknown node. With a determined anchor node, the RSSI algorithm can be used for the localization. The new RSSI localization algorithm satisfies strict requirements on localization and reaches higher levels of short distance measurement accuracy. The measured distance between the unknown node and the anchor nodeis generally noted as $\mathrm{D}_{\mathrm{i}}$. The variable is usually initialized as 0 . After determining the distance between the unknown node and the anchor node, the researcher should measure the incident angle between the two nodes. The measurement mainly relies on the AOA algorithm. To ensure the accuracy, the angle is normally measured in the counter-clockwise direction and the result is saved as $i$.

(3) After all the information is processed, the researcher should perform geometric calculation to get the coordinates of the unknown node.

$$
\left\{\begin{array}{l}
x_{p i}=x_{i}+\cos \theta_{i} D_{i} \\
y_{p i}=y_{i}+\sin \theta_{i} D_{i}
\end{array}\right.
$$

Obtaining the coordinates of the unknown node, the researcher needs to convert the node into an anchor node. Some of the parameter values in the packet are fixed, while the value of $A_{i d}$ would change. If the value is 1 , it can be concluded that the reference anchor node used to localize this node is embedded in the system; if the value is 2 , the reference anchor node should be deemed as a level 2 node. Hence, the value of $A_{i d}$ determines which type of anchor node the unknown node would be converted into. After conversion, change the hop count back to 0 , save the coordinates of the corresponding node, and thereby obtain the data packet of the new anchor node.'

(4) After it is determined, the new anchor node would broadcast information to the unknown nodes within its own communication coverage and, at the same time, send the data packets it receives. Then, the researcher should convert the corresponding unknown node. In this way, all the qualified anchor nodes would be obtained. The localization would be complete when the entire network is traversed.

\subsection{The flowchart of the improved algorithm}

The function of the improved algorithm has also undergone great changes. See Figure 3 for the flowchart. The improved algorithm achieves very high network coverage by making use of the broadcast function of the original algorithm. The most striking feature of the algorithm is the addition of multiple new nodes, which dramatically expands the localization range.

\section{$4 \quad$ Algorithm simulation and result analysis}

For the purpose of verifying the performance of the improved localization algorithm proposed in this paper, the author employs mathematical experiment simulation software to perform a simulation analysis. The software is extensively used because it is capable of simulating the WSN environment and identifying the localization accuracy of the target algorithm. In light the simulation results, it is possible to select a proper topology, and compare and analyze the performance of different localization 
algorithms. On this basis, the algorithm can be improved to get higher localization accuracy.

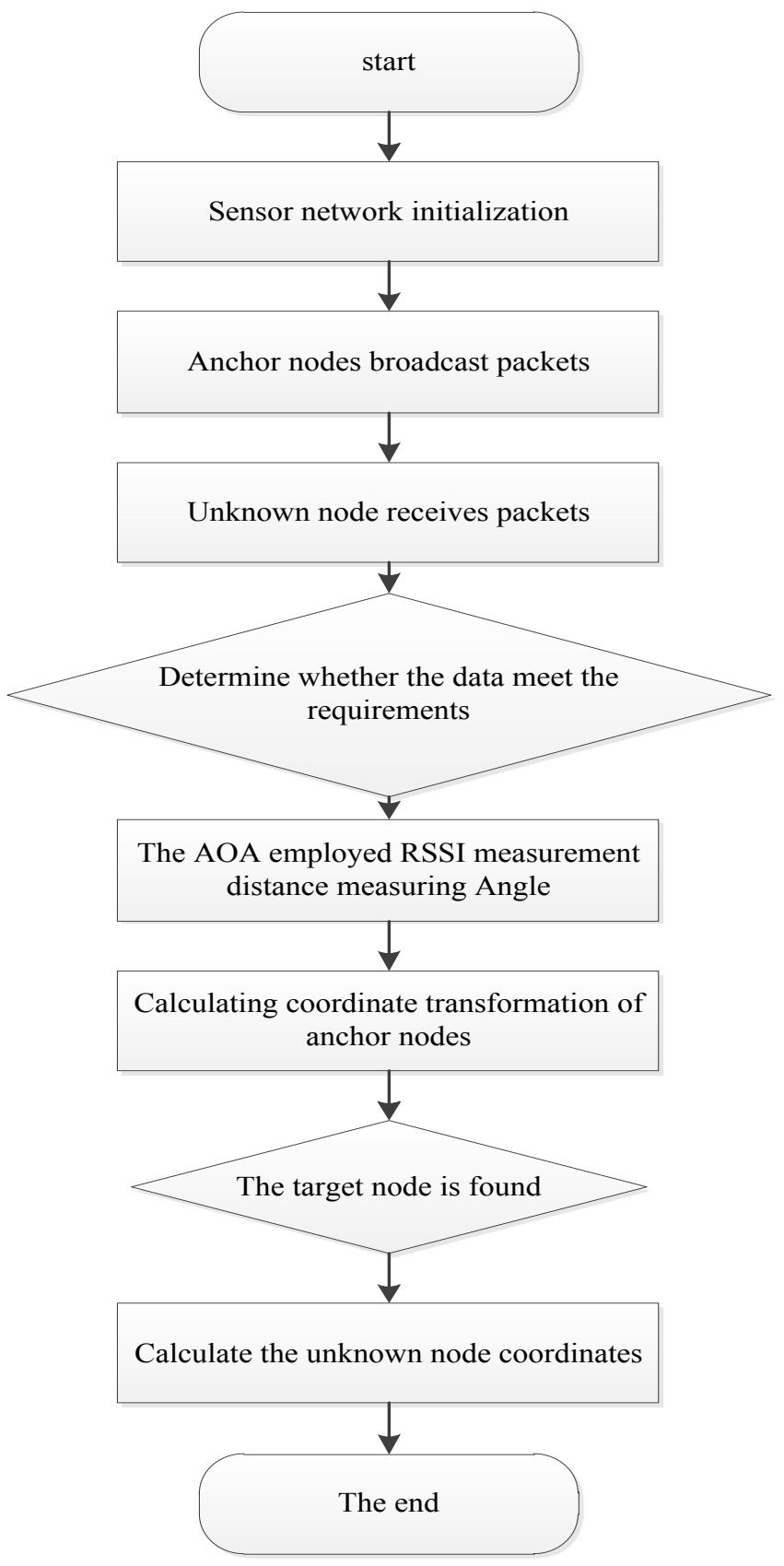

Fig. 3. The flowchart of the improved algorithm 


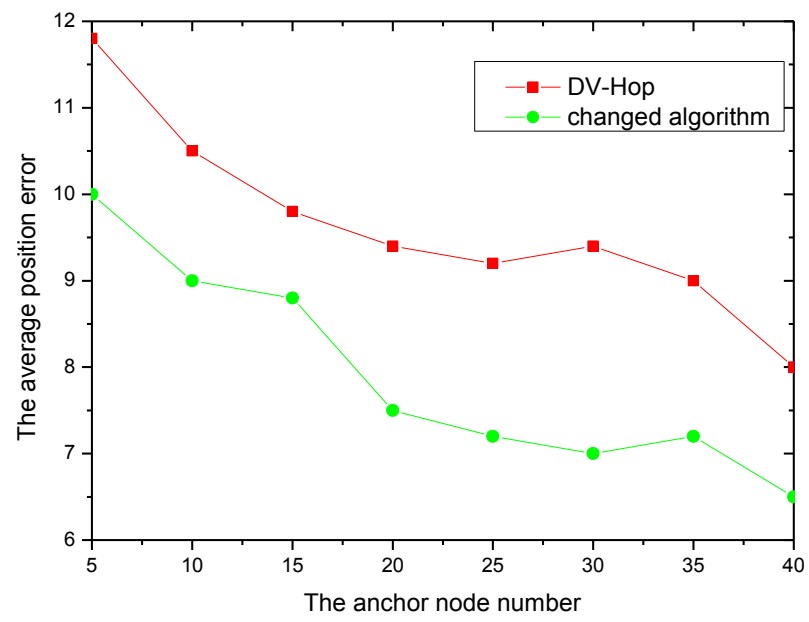

Fig. 4. Comparing the errors of different numbers of anchor nodes

(1) Taking different numbers of anchor nodes

Based on Figure 4, it can be seen that the two algorithms discussed in this paper have basically unchanged network parameters and the only difference between them lies in the changing values of the anchor node. In this figure, the number of anchor nodes falls between 5 and 40 and the step size is 5 . If the total number of nodes in the network remains the same, the localization accuracy of both of the algorithms would rise in different degrees, resulting in lower error rate, as the number of anchor nodes increases. Nevertheless, on the whole, the improved algorithm is more powerful than the traditional one, featuring much better localization accuracy.

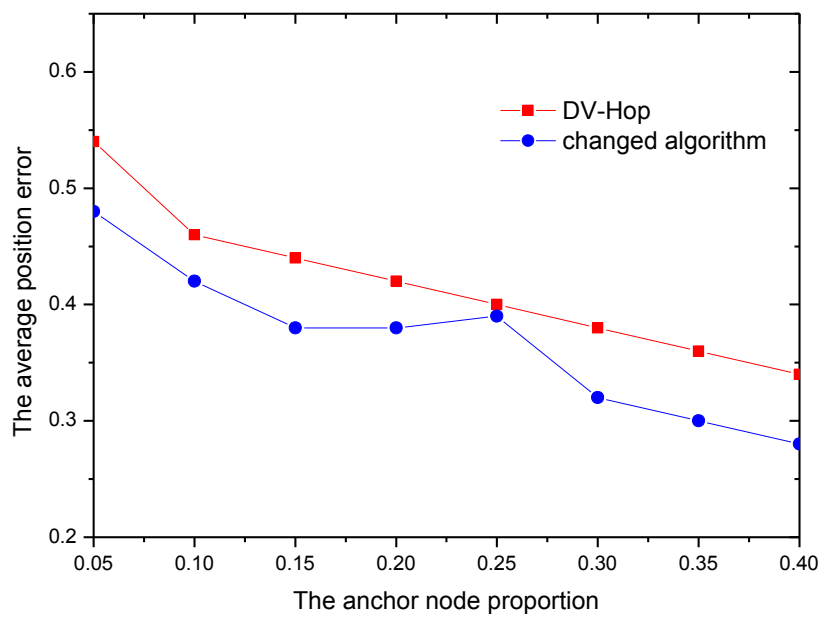

Fig. 5. Communication radius $\mathrm{R}=5 \mathrm{~W}$ 
(2) Simulation of different communication radiuses

This section talks about the simulation carried within different communication radiuses. Figures 5, $6 \& 7$ display the simulation results at three different communication radiuses: $\mathrm{R}=15 \mathrm{~m}, 20 \mathrm{~m}$ and $30 \mathrm{~m}$. In these figures, the $\mathrm{x}$-axis stands for the ratio of the number of anchor nodes to the total number of network nodes, while the $y$-axis represents the average localization errors of the two algorithms. Comparing the three figures, the author discovers that the number of anchor nodes is closely related to the accuracy of the algorithms. With the increase of the number of the nodes, both algorithms have higher localization accuracies and lower error rates. Besides, the error of the improved algorithm decreases more significantly than that of the other algorithm. Further comparison indicates that, when the communication radius is $15 \mathrm{~m}$, the error of the improved algorithm is $2 \%-15 \%$ smaller than that of the traditional algorithm; when the communication radius is $20 \mathrm{~m}$, the error of the improved algorithm is $2 \%$ $7 \%$ smaller than that of the traditional algorithm; when the communication radius is further increased to $30 \mathrm{~m}$, the error of the improved algorithm is $2 \%-10 \%$ smaller than that of the traditional algorithm. The statistics reveal that the improved algorithm is much more accurate than the traditional one within short distances. See Figure 8 for the specific localization accuracy of the improved algorithm at different communication radiuses. It is clearly described in the figure that there is a certain correlation between the communication radius and the localization error. As the communication radius increases, the algorithm has an increasing high accuracy and increasingly low error value. As a result, the communication error can be reduced by lengthening the communication radius.

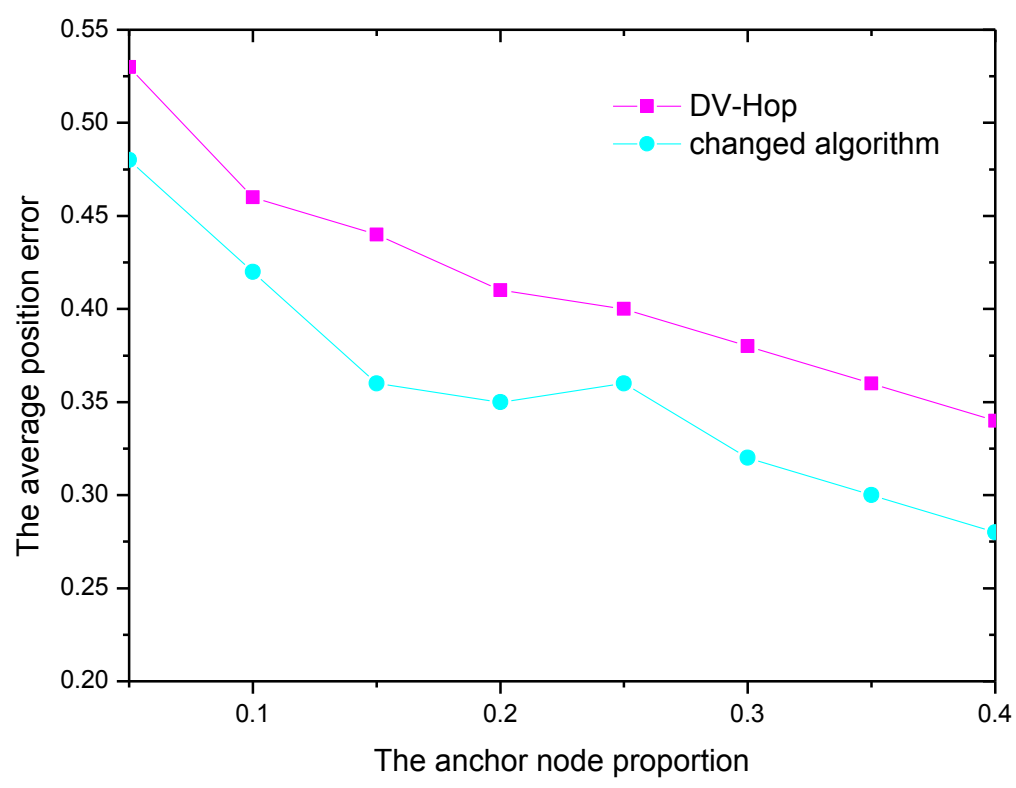

Fig. 6. Communication radius $\mathrm{R}=20 \mathrm{~W}$ 


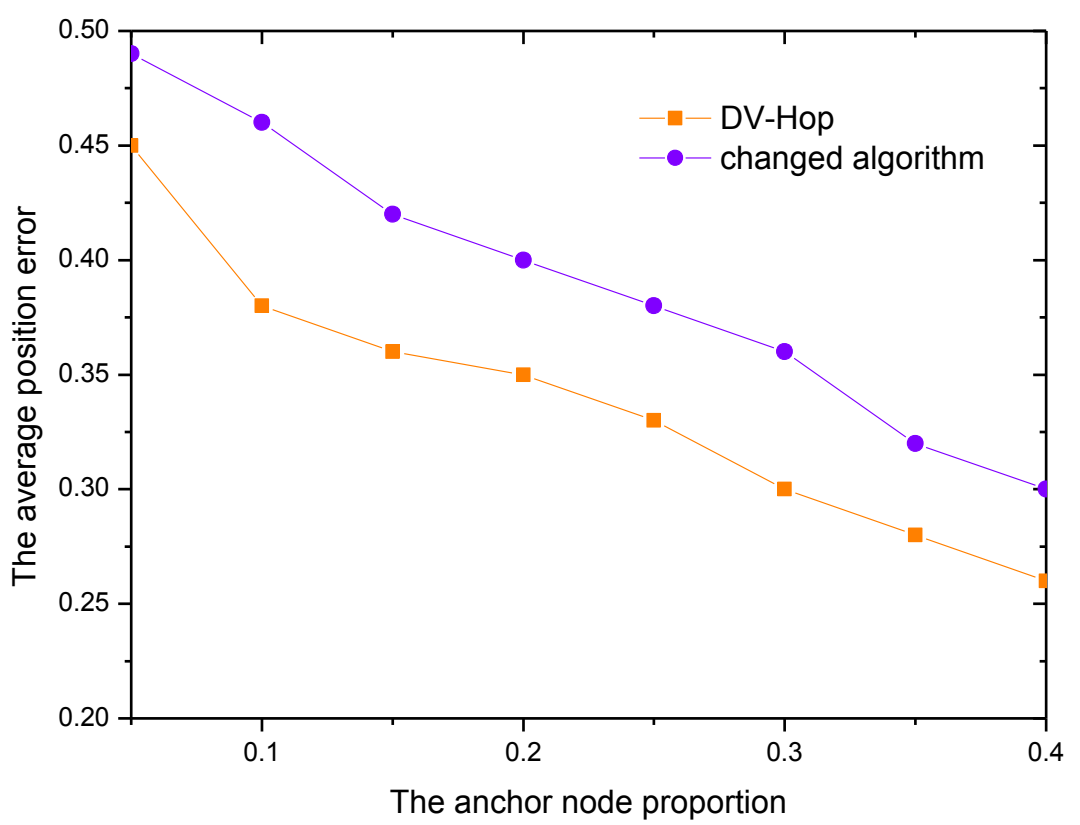

Fig. 7. Communication radius $\mathrm{R}=30 \mathrm{~W}$

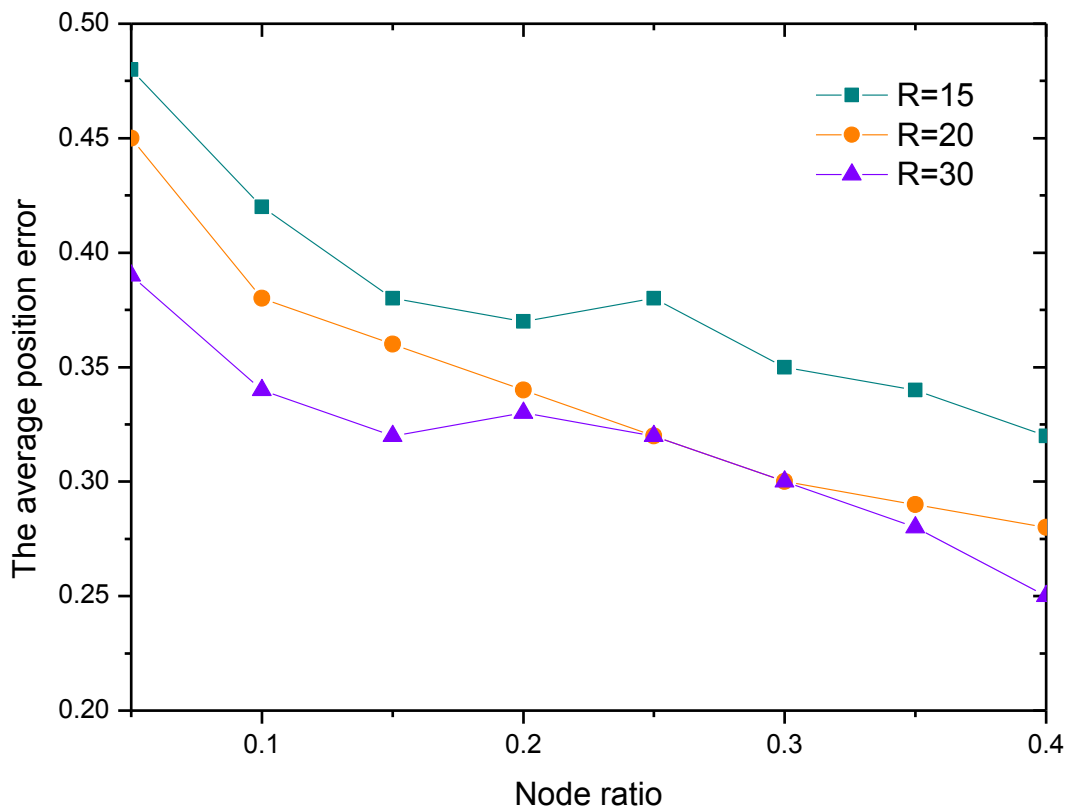

Fig. 8. The performance of the improved algorithm at different communication radiuses 


\section{Conclusion}

This paper mainly improves the traditional DV-Hop algorithm, and proposes a new node localization algorithm based on one-hop range. After that, the author carries out in-depth analysis of the principles and performance of the two algorithms and verifies the performance by simulation. To sum up, this paper mainly deals with the following issues:

(1) Through the analysis of the localization principle of the algorithm, the author holds that it is possible to measure the spacing between anchor node and unknown node via RSSI technology. In accordance with the requirements on specific distance localization, the author reaches the conclusion that the RSSI helps reduce the measurement error.

(2) The author introduces the AOA measurement technology. The technology improves the accuracy of weapon attack because it can easily determine the orientation of anchor node, and identify the incident angle between the two nodes.

(3) This paper mainly localizes the nodes by the mathematical method so that the data can be easily processed in a computer. In this way, the calculation error is reduced without sacrificing the calculation speed.

(4) In the meantime, this paper uses anchor nodes to assist in localization. If an unknown node has been localized, it can be used to help with the localization of other unknown nodes. Since some nodes are far away from or beyond the measuring range of the anchor node, it is necessary to increase the number of anchor nodes to improve the coverage of the network, thereby fulfilling the monitoring purposes.

(5) The author selects the priority method to determine the appropriate anchor nodes, and transforms unknown nodes according to the existing anchor nodes. Besides, the author classifies the nodes into different levels, and chooses the most prioritized anchor nodes to help with the localization.

(6) Finally, the author simulates the two algorithms, compares the measuring accuracies and errors between the two, and provides the flowchart and complier language of the new algorithm.

\section{Acknowledgment}

Thanks very much for $12^{\text {th }}$ Five Year National Science and Technology Support Plan (Grant No.: 2014BAD06B03), the Science and Technology Development Program of Jilin Province (Grant No.: 20160441004SC). These financial supports are gratefully acknowledged.

\section{$7 \quad$ References}

[1] Aydin, I., Karaköse, M. (2013). Wireless sensor network based fault diagnosis approaches, 201321 st Signal Processing and Communications Applications Conference, 201-211. 
Paper-On the Localization Algorithm of Wireless Sensor Network and Its Application

[2] Li, J. Y., Wang, J. P. (2014). Wireless sensor network mobile agent routing based on the improved ant colony algorithm. Journal of Convergence Information Technology, 8(5), 585-592. https://doi.org/10.4028/www.scientific.net/amm.587-589.2339

[3] Aktouf, O. E. K., Parissis, I. (2012). SMART service for fault diagnosis in wireless sensor networks, Proceedings - 6th International Conference on Next Generation Mobile Applications. 211-216.

[4] Lu, T. J., Wang, Y. (2014). Application of improved ant colony algorithm technology in development of routing protocol in wireless sensor network. Applied Mechanics \& Materials, 685, 583-586. https://doi.org/10.4028/www.scientific.net/AMM.685.583

[5] Li, T., Fei, M. (2010). Fault diagnosis of auxiliaries in power plants based on wireless sensor networks with vibration transducer. IEEE International Conference on Network Infrastructure and Digital Content, IC-NIDC 2010, 732-736. https://doi.org/10.1109/ICNIDC. 2010.5657877

[6] Tang, B., Deng, B., Deng, L. (2016). Mechanical fault diagnosis method based on multilevel fusion in wireless sensor networks, Zhendong Ceshi Yu Zhenduan/Journal of Vibration, Measurement and Diagnosis, 36, 92-96.

[7] Lia, M. H., Hua, Z., Guang, S. (2012). Energy aware routing algorithm for wireless sensor network based on ant colony principle. Journal of Convergence Information Technology. Journal of Convergence Information Technology, 7, 215-221. https://doi.org/10.4156/jcit. vol7.issue4.26

[8] Zhong, J. H., Zhang, J. (2012). Ant colony optimization algorithm for lifetime maximization in wireless sensor network with mobile sink. Conference on Genetic and Evolutionary Computation, 18(12), 161-166.

[9] Khilar, P. M., Mahapatra, S. (2007). Intermittent Fault Diagnosis in Wireless Sensor Networks. International Conference on Information Technology ( 145-147). IEEE Computer Society, 145-147.

[10] Li, Q. (2013). Wireless sensor network fault diagnosis method of optimization research and simulation. Applied Mechanics \& Materials, 347-350, 955-959. https://doi.org/10.4028/www.scientific.net/AMM.347-350.955

[11] Yao, Y. C., Yao, Y. (2013). The application of ant colony optimization in wireless sensor network routing. Advanced Materials Research, 655-657, 838-841. https://doi.org/10.4028/www.scientific.net/AMR.655-657.838

[12] Zhang, J. Y., Chen, D. Y. (2014). Clustering routing algorithm ant colony optimizationbased for wireless sensor network. Applied Mechanics \& Materials, 568-570, 594-597. https://doi.org/10.4028/www.scientific.net/amm.568-570.594

[13] Tian, J., Gao, M., Ge, G. (2016). Wireless sensor network node optimal coverage based on improved genetic algorithm and binary ant colony algorithm. EURASIP Journal on Wireless Communications and Networking, 2016(1), 236-239. https://doi.org/10.1186/s13638016-0605-5

[14] Wu, X., Qi, L. (2013). The optimization algorithm of wireless sensor network node based on improved ant colony, Sensors and Transducers, 155, 54-63.

[15] Chang, S. H., Merabti, M., Mokhtar, H. (2010). A Causal Model Method for Fault Diagnosis in Wireless Sensor Networks. IEEE International Conference on Computer and Information Technology, CIT-2010, 155-162. https://doi.org/10.1109/CIT.2010.65

[16] He, C. L. (2013). Technology research on the fault diagnosis of wireless sensor network system. Advanced Materials Research, 846-847(267), 442-445. https://doi.org/10.4028/ www.scientific.net/AMR.846-847.442 
[17] Jin, X., Chow, T. W., Sun, Y., Shan, J., Lau, B. C. (2015). Kuiper test and autoregressive model-based approach for wireless sensor network fault diagnosis. Wireless Networks, 21(3), 829-839. https://doi.org/10.1007/s11276-014-0820-0

[18] Liu, R. F. (2010). Fault diagnosis of wireless sensor based on ACO-RBF neural network, Proceedings-2010 3rd IEEE International Conference on Computer Science and Information Technology, ICCSIT 2010, 248-251.

\section{Authors}

Honglei Jia is a professor of Jilin University, and he is also a member of Chinese Agricultural Mechanical Association. His research interests are Agricultural Mechanization Engineering and Conservation Tillage. He is affiliated to College of Biological and Agricultural Engineering, Jilin University, Changchun 130022, China and the Key Laboratory of Bionic Engineering, Ministry of Education, Jilin University, Changchun 130022, China (jiahl@vip.163.com).

Jiaxin Zheng is a doctoral student of Jilin University whose major is Agricultural Mechanization Engineering and Conservation Tillage. Affiliated to the College of Biological and Agricultural Engineering, Jilin University, Changchun 130022, China and the Key Laboratory of Bionic Engineering, Ministry of Education, Jilin University, Changchun 130022, China (zhengjiaxin_0628@126.com).

Gang Wang is a postdoctor in Electrical Science and Engineering of Jilin University. Affiliated to the College of Biological and Agricultural Engineering, Jilin University, Changchun 130022, China and the Key Laboratory of Bionic Engineering, Ministry of Education, Jilin University, Changchun 130022, China (wangganggoodman@126.com).

Yulong Chen is a doctoral student of Jilin University whose major is Agricultural Mechanization Engineering and Conservation Tillage. Affiliated to the College of Biological and Agricultural Engineering, Jilin University, Changchun 130022, China and the Key Laboratory of Bionic Engineering, Ministry of Education, Jilin University, Changchun 130022, China (1097402292@qq.com).

Dongyan Huang is a Professor in Jilin University. His research interests include Mechanical Design, Automatic Control and Computer Application Technology. Affiliated to the College of Biological and Agricultural Engineering, Jilin University, Changchun 130022, China and the Key Laboratory of Bionic Engineering, Ministry of Education, Jilin University, Changchun 130022, China (cchdy760829@sina.com).

Hongfang Yuan is a postdoctor in Electrical Science and Engineering of Jilin University. Mainly engaged in study on Conservation Tillage Technique and Agriculture Mechanization Design. Affiliated to the College of Biological and Agricultural Engineering, Jilin University, Changchun 130022, China and the Key Laboratory of Bionic Engineering, Ministry of Education, Jilin University, Changchun 130022, China (yhf1984828@163.com).

Article submitted 09 Febraury 2017. Published as resubmitted by the authors 14 March 2017. 